\title{
Rabaska
}

Revue d'ethnologie de l'Amérique française

\section{DELISLE, JEAN. Interprètes au pays du castor. Québec, Presses de l’Université Laval, 2019, 374 p. ISBN 978-2-637-4653-1}

\section{Gilles Havard}

Volume 18, 2020

URI : https://id.erudit.org/iderudit/1072931ar

DOI : https://doi.org/10.7202/1072931ar

Aller au sommaire du numéro

Éditeur(s)

Société québécoise d'ethnologie

ISSN

1703-7433 (imprimé)

1916-7350 (numérique)

Découvrir la revue

Citer ce compte rendu

Havard, G. (2020). Compte rendu de [DELISLE, JEAN. Interprètes au pays du castor. Québec, Presses de l'Université Laval, 2019, 374 p.

ISBN 978-2-637-4653-1]. Rabaska, 18, 334-336. https://doi.org/10.7202/1072931ar d'utilisation que vous pouvez consulter en ligne.

https://apropos.erudit.org/fr/usagers/politique-dutilisation/ 
(Atikamekw), Pagwajininiwag ou le Wendigo, de même que les jongleurs (Algonquins). Chaque recueil a une section consacrée aux " Animaux », parfois jumelés à des plantes : le carcajou, le lièvre peureux, le porc-épic, le caribou bicéphale, l'aigle géant, souvent le castor, qui est très apprécié pour la saveur de sa viande, la petite plante et le petit garçon. La section «Au contact d'autres nations » traite en particulier des rapports avec l'autre et de la défense des territoires ancestraux, menacés par les intrusions des étrangers souvent sans scrupule. On y trouve, par exemple, les contacts souvent meurtriers avec les Iroquois et une belle version de « La Légende de la chute aux Iroquois » (Les Algonquins). Au cas où des récits auraient été oubliés, chaque recueil se termine par ce que l'auteur a appelé des « Récits divers ». Il s'agit d'histoires se rapportant à quelques êtres peuplant l'environnement, des voleurs de nourriture, des personnages souvent loufoques, ou évoquant entre autres le rêve, parfois des contes éducatifs ou mnémotechniques consacrés à la flore (Les Algonquins).

À la fin de chaque recueil, s'étendant sur plusieurs pages, Clément fournit, sous le titre « Sources et Notes », une foule d'informations sur la provenance de chacun des récits sélectionnés, sur les collecteurs qu'il identifie, sur le contexte de la cueillette, en plus de livrer d'utiles renseignements sur la spécificité de plusieurs récits, rappelant les us, coutumes et traditions des communautés, sans oublier, à l'occasion, de noter les variantes avec d'autres récits. Voilà certes de quoi réjouir les spécialistes, tout comme les bibliographies qui clôturent chaque recueil.

La lecture de ces récits m'a comblé et m'a permis d'entrer en contact avec ces peuples que souvent nous ne connaissons pas ou si peu. Un souhait pour la suite de la série : l'auteur ne pourrait-il pas ajouter quelques notes infrapaginales pour expliquer certains mots indiens et certaines expressions, telle la « tente tremblante », par exemple, phénomène qui se produit dans ces communautés mais que les non-spécialistes comme moi ne connaissent pas. Voilà qui serait utile à plusieurs lecteurs.

Aurélien Boivin

Université Laval (Québec)

Delisle, Jean. Interprètes au pays du castor. Québec, Presses de l'Université Laval, 2019, 374 p. ISBN 978-2-637-4653-1.

Les historiens de la rencontre entre Euro-Américains et autochtones au Canada ont souvent négligé les phénomènes linguistiques. De ce point de vue, l'ouvrage de Jean Delisle, professeur émérite de l'Université d'Ottawa et spécialiste des questions de traduction, est le bienvenu, puisqu'il nous plonge 
dans l'univers des interprètes, ces médiateurs entre les cultures et traducteurs de mondes. L'auteur ne propose certes pas une histoire sociale « totale » des interprètes du Canada ou de la Nouvelle-France continentale (un type de recherche qui reste à mener). Privilégiant la méthode biographique, il brosse le portrait d'une quinzaine de truchements, un (voire deux) par chapitre, dont certains occupent aujourd'hui une petite place dans la mémoire canadienne : cinq des personnages présentés ici, Nicolas Perrot, Tookoolito, Ebierbing, Étienne Brûlé et Thanadelthur, ont ainsi été honorés par le gouvernement canadien au rang de «personne d'importance historique nationale» (p. 325). Dans l'ensemble, Delisle redonne vie à des obscurs, des sans-voix, qui ne surgissent dans l'histoire (sauf pour quelques manieurs de plume) qu'à la faveur des récits de leurs employeurs ou de relateurs les ayant rencontrés. Le parti-pris biographique lui permet de privilégier une écriture narrative. Il lui ôte peut-être aussi l'envie ou la tentation d'explorer pleinement les sources disponibles. À cet égard, l'appareil de notes se révèle minimaliste.

L'intérêt du livre est d'offrir des portraits variés - des hommes et des femmes, des Euro-Américains, des Amérindiens, des Inuits et des Métis et d'adopter une perspective élargie : chronologique, l'ouvrage embrasse l'ensemble du Canada contemporain, de l'Acadie aux Rocheuses (en laissant de côté la vallée du Mississippi), et une période s'étirant du début du XvI ${ }^{\mathrm{e}}$ à la fin du XIX ${ }^{\mathrm{e}}$ siècle. L'auteur souligne ainsi la profondeur historique et géographique du phénomène canadien des truchements - l'absence d'un véritable appareil de cartes est à cet égard regrettable. Delisle nous met aussi en garde contre l'anachronisme, par exemple à propos de l'interprète d'origine africaine Mathieu Da Costa (chap. 2), que certains ont pu qualifier de façon grossière de « père du multiculturalisme » canadien (p. 41). Laissant de côté les missionnaires, peut-être parce qu'ils sont les plus étudiés, il propose un classement des interprètes en cinq catégories : compagnon des explorateurs (tels Domagaya et Taignoagny, truchements iroquoiens de Jacques Cartier); collaborateur des autorités civiles (tel Nicolas Perrot) ; officier militaire (tel Chabert de Joncaire) ; trafiquant ou émissaire de compagnies de fourrures (tel Étienne Brûlé) ; enfin, aide-missionnaire (tel Jean L'Heureux, chez les Pieds Noirs). L'une des originalités d'Interprètes au pays du castor est de prendre en considération des cas d'interprètes amérindiens, inuits et métis. "Bien qu'ils soient mentionnés dans les journaux des explorateurs, ils demeurent cantonnés dans les marges de l'histoire ", écrit Delisle dans le chapitre consacré à Tattannoeuck, l'Inuit qui a guidé John Franklin lors de ses explorations arctiques (p. 179). L'auteur met aussi en valeur la variété des rôles joués par les interprètes : truchement, guide, négociateur et même, écrit-il, « anthropologue » (p. 49 ; en réalité, il s'agissait au mieux d'ethnographes). L'ouvrage, curieusement, se penche assez peu sur les contingences de la 
traduction. De ce point de vue, quelques travaux importants échappent à la sagacité de l'auteur, tels la thèse (et les nombreux articles) du linguiste Robert Vézina, ou les ouvrages de l'historien Edward G. Gray (New World Babel. Language \& Nations in Early America; The Language Encounter in the Americas, 1492-1800). À la lecture des parcours biographiques, on est aussi en droit de se demander si les personnages choisis sont des cas d'école ou si leurs trajectoires sont exceptionnelles. C'est la seconde réponse qui émerge - Delisle parle «d'interprètes d'exception » (p. 5), mais on aimerait en savoir un peu plus sur leur représentativité.

Grisé par ses personnages, l'auteur succombe parfois à une veine héroïque ou aux images d'Épinal. Il prête aussi à ses héros des traits physiques ou des caractéristiques psychologiques qui mériteraient davantage de références documentaires : "selon des témoignages, les trois filles de Pierre Couc se distinguent par leur grande beauté et leur vivacité d'esprit »; "ils [Élisabeth Couc et le chef outaouais Outoutagan] tombent éperdument amoureux » (p. 140-142), etc. Qu'en sait-on au juste ? Comme l'ont montré les travaux minutieux de Suzanne Boivin Sommerville, l'historiographie a souvent insufflé de la romance dans la vie d'Isabelle/Élisabeth Couc, au prix de la rigueur. C'est lorsque l'auteur - par exemple dans les chapitres consacrés à Joncaire, à John Long et à John Tanner - prend davantage en compte la question des sources que l'ouvrage devient le plus intéressant. Mais pourquoi, par ailleurs, continuer à faire de Nicolas Perrot un « donné » des jésuites, alors qu'aucune source ne l'indique ? L'utilisation de travaux récents sur Étienne Brûlé aurait aussi permis de nuancer certaines affirmations. L'auteur, de surcroît, se montre assez peu sensible aux recherches ethnohistoriques et anthropologiques. Quand il écrit par exemple que Nicolas Perrot « réussit [en 1671] à convaincre ces peuples [les autochtones des Grands Lacs] de devenir sujets du Roi Soleil » (p. 73), il pose une équivalence entre le discours colonial, la réalité des relations franco-indiennes et la perception des autochtones qui s'avère illusoire. L'auteur manque aussi de recul vis-à-vis des sources quand, à la manière des anciens observateurs, il dépeint les femmes amérindiennes comme de « véritables bêtes de somme » (p. 90).

Malgré ces limites, le livre a le mérite d'explorer une thématique peu traitée dans l'historiographie canadienne et de le faire à travers une juxtaposition de récits de vie susceptibles de plaire à un large public.

Gilles Havard

CNRs, Paris 